\title{
EFFECTS OF HIGH DOSE COFFEE INTAKE ON AEROBIC POWER IN DRAGON FEMALE ATHLETES
}

Ramin Shabani, Roya Rahmatian, Fatemeh Izaddoust

Department of Exercise Physiology, Rasht Branch, Islamic Azad University, Rasht, Iran

\begin{abstract}
Background: There are few studies that consider the effect of high doses of caffeine on aerobic power (VO2max). Also, to date, no study examined the effect of coffee intake on dragon boat paddler specifically on women. The purpose of this study was to investigate the effect of espresso coffee on improvement of aerobic power of dragon boat paddler. Material: Twenty women athletes of Guilan dragon bout team members of Malavan club of port city of Anzali (mean \pm SD age, $23.60 \pm 3.49$ years; BMI, $23.77 \pm 1.88 \mathrm{~kg} / \mathrm{m}^{2}$; body fat, $30.32 \pm 4.65 \%$ ) were recruited to this study, after they completed a primary test without consuming any coffee, they consumed $6 \mathrm{mg} / \mathrm{kg}$ of coffee (espresso or decaffeinated) and following that they completed two experimental trials. A randomized, double-blind, repeated-measures, design was employed whereby paddlers complete a $2000 \mathrm{~m}$ paddling dragon boat ergo-meter. Results: Coffee could improve VO2max (Without coffee $=74.40 \pm 4.99$, Espresso coffee $=90.10 \pm 6.19$, Decaffeinated coffee $=91.00 \pm 5.67, \mathrm{P} \leq 0.05)$. VO2max amount after exercise were significantly higher for both espresso coffee and decaffeinated coffee, when compared with without coffee condition. No significant differences were observed between espresso coffee and decaffeinated coffee $(\mathrm{P} \leq 0.05)$. Conclusion: The present study shows that both high doses of caffeine (espresso coffee) and decaffeinated coffee can enhance VO2max during aerobic exercise including $2000 \mathrm{~m}$ dragon boat paddling. It seems that some compounds except caffeine in decaffeinated coffee can act improve VO2max. Further studies needed for considering the effect of high doses of coffee on endurance exercises. Also in other age ranges of women athletes and other sport athletes.
\end{abstract}

Keywords: caffeine, exercise, athletes, boating, VO2max.

\section{Introduction}

Dragon boat, a team paddling water sport, has developed in nearly 60 countries worldwide. Also annually over 50 million people in Asia are participant in dragon boat competitions. Dragon boat races are from 200 to 2000 meters and above. Cardiovascular endurance(In terms of VO2max) is one of the important skills for dragon boat paddlers [1] and a dragon boat paddler should ideally be able to maintain VO2max throughout the whole game $[2]$.

Coffee contains mineral ingredients such as $\mathrm{Ca}, \mathrm{K}, \mathrm{Fe}, \mathrm{P}, \mathrm{Ni}, \mathrm{Mg}$, and $\mathrm{Cr}$ [3], polyphenols, melanoidin and caffeine [4]. Numerous studies have shown that caffeine can act as an ergo-genic aid, in long-term exercise [5-9]. Caffeine's primary ergo-genic mode of action is on the CNS [10]. Also it can be energetic with an increase in fat oxidation and a decrease in glycogen use [11]. Caffeine switches the substrate preference from glycogen to fat by increasing hormone sensitive lipase (HSL) activity and inhibition of glycogen phosphorylase activity [12].

Most research, have investigated the effect of low or moderate doses of caffeine consumption on performance, perceptual responses and Neuromuscular factors prior to endurance exercise [7-9,13, 14]. Nevertheless, there are a few studies that considered the effect of high doses of caffeine on endurance exercise $[15,16]$. This may seem surprising as reports have shown that coffee is the most concentrated dietary source of caffeine as well as being one of the largest sources of caffeine used by athletes prior to competition [17]. However, according to our information to date, no study examined the effect of coffee ingestion on dragon boat paddler specifically on females.

In addition, a few studies indicated the effect of pre exercise coffee on endurance exercises $[7,8,16,18$ 20] and they demonstrated difference results. Hodgson et al. (2013) utilized coffee and caffeine in their Research and shown that both of caffeine and coffee $(5 \mathrm{mg} / \mathrm{kg})$ consumption one hour prior to exercise can improve endurance exercise performance [8]. In contrast, Graham et al. (1998) Resulted that caffeine capsule could increase endurance compare to placebo but coffee don't have any effect on endurance [19]. In another study by Graham et al. (1995) they showed endurance enhanced with both 3 and $6 \mathrm{mg} / \mathrm{kg}$ of caffeine, whereas there was no significant effect with $9 \mathrm{mg} / \mathrm{kg}$ of caffeine [16]. Moreover, to our knowledge, there is the first study that considered the ergogenic effect of espresso coffee on aerobic performance compared to decaffeinated coffee or placebo.

Therefore, the purpose of this study was to determine the effects of high dose coffee (espresso coffee) on $2000 \mathrm{~m}$ dragon boat women paddler compared to decaffeinated coffee or placebo.

(c) Ramin Shabani, Roya Rahmatian,

Fatemeh Izaddoust, 2016

doi:10.15561/20755279.2016.0406 


\section{Materials and Methods \\ Subjects \\ Participants.}

Twenty women athletes of Guilan dragon bout team members of Malavan club of port city of Anzali (mean \pm SD age, $23.60 \pm 3.49$ years; BMI, $23.77 \pm 1.88 \mathrm{~kg} / \mathrm{m}^{2}$; body fat, $30.32 \pm 4.65 \%$ ) with at least third time per week engaged in training and had competitive paddling experience were recruited for the study. The experiments were performed within the period of training phase. All participants didn't have any injuries and they were non-smokers. For inclusion in the study, volunteers should not use other nutritional supplements. All athletes were fully informed of the experimental trials and all risks and discomforts associated before providing written informed consent to participate in the study.

Ethical approval was obtained from the Islamic Azad University Rasht Branch Ethics Committee.

Research Design.

Method: The study utilized a double-blind, repeated-measures, design to identify the effects of espresso coffee and decaffeinated coffee on VO2max changes (2000m paddling). Research Subjects were asked to attend three laboratory sessions for doing two trails with coffee ingestion and one trail without coffee. All they took part in first session and two other sessions conducted 2 week apart and being performed at the same time in the morning to reduce the potential for circadian variability. In the first session participants pre-test measurements were assessed. Then they were performed the test without coffee and Subjects were given a questionnaire that measured feeling of perceived fatigue [21]. During the following two other visits, the subjects intake either the espresso coffee or a decaffeinated coffee and then remained seated for 60 minutes before the test. Athletes initially warmed up on a Dragon Boat Ergo-meter (D1-M) with the desired intensity and speed for $10 \mathrm{~min}$. Then rested for 2 minute before the starting the tests. Then they performed 2000 meters paddling on a dragon boat ergo-meter that was calibrated prior each test by the athlete weight. Two second and third trails were separated by $24 \mathrm{~h}$ and the VO2max was recorded at the end of each test (showed by ergo-meter monitor). A Dragon Boat trainer that was Knows how worked a Dragon Ergo-meter and also was blind to the study protocol, supervised all sessions. Subjects were asked to maintain their habitual dietary intake and to abstain from coffee or vigorous physical activity for $24 \mathrm{~h}$ prior to each test. Also, all participants had previous experience with a dragon boat ergo-meter.

Pre-test assessment: Height and body weight (lightly dressed and barefooted) were measured to the nearest $0.1 \mathrm{~cm}$ nearest $0.01 \mathrm{~kg}$, respectively, using a stadiometer and scale (Seca - Germany). Skinfold thicknesses were obtained at 3 sites (Triceps, Suprailiac, Thigh) according to the Standardization Reference Manual [22] using a Saehan caliper (SH5020, Korea), and body fat percentage were estimated using Jackson and Pollock nomogram. Body mass index were assessed using weight/height ${ }^{2}$ (Quetelet, 1869).

Perceived fatigue: The perception of fatigue was performed using a Borg scale. The subjects indicated their own perception of fatigue immediately after the protocol on a scale of 0 to 10 . Zero indicated least fatigue, and the ten indicated most fatigue.

Aerobic power assessment: Kayakers performed their 2000m test on ergo-meter task specificity trial on an air braked, drag adjustable Multistroke Dragon Boat Ergo-meter (D1-M). The ergo-meter consisted of a flywheel connected to a carbon shaft via a retractable cord attached at either end. Distance from seat to foot-bar was adjusted to match each individual's seat position in the kayak; hand position on the carbon shaft was also adjusted to match on-water paddling position. Paddler's weight in $\mathrm{Kg}$ was inputted in board console then accelerated the fan to a speed of 700 or over. Once the acceleration / speed is above 700, Was allowed the flywheel run freely until the fan had come to an unimpeded or un-accelerated stop, then the Drag Factor was automatically set. This calibration was performed prior to each test for all athletes according to manufacturer's recommendations. Subjects were performed $2000 \mathrm{~m}$ paddling (determined by the covered distance was showed on monitor) in each session and one of the author's was supervised all sessions which was blind. VO2max were calculated at the end of each trail using the Dragon Boat ergo-meter, showed on ergo-meter monitor [23].

Coffee: three exercise trials were performed under three conditions, one with espresso coffee, the other one with decaffeinated coffee and third performed without any coffee. Subject ingested $6 \mathrm{mg}$ caffeine $/ \mathrm{kg}$ of espresso coffee, a range of 500-575 mg/kg, (based on a content of $145 \mathrm{mg}$ caffeine $/ 100 \mathrm{~g}$ coffee, Nestlé- NESCAFE) or 6 $\mathrm{mg}$ caffeine/kg of decaffeinated coffee (caffeine amount was negligible, Nestlé-NESCAFE) 60 minutes before the test [24]. Amount of Coffee needed was measured using electronic weighing machine (Sartorius GMBH by Gottingen Germany). Both espresso coffee and decaffeinated coffee were dissolved in $200 \mathrm{ml}$ hot water and Not added any sweetener.

Statistical Analysis 
Values are expressed as means \pm SD. The normality of each data set was evaluated using the KolmogorovSmirnov test, which confirmed a normal distribution. Results were analysed using the general linear model with repeated measures. Where significant main effects were observed, Bonferroni post hoc tests were used to determine the differences. All data were analyzed using IBM SPSS Statistics version 21 for Windows (SPSS Inc., Chicago, IL, USA). A P value of less than 0.05 was considered to be statistically significant.

\section{Results}

Participants' $(\mathrm{n}=20)$ descriptive statistics include age, weight, height, BMI, body fat percent and Borg perceived fatigue are listed (Table 1).

Table1. Participant characteristics $(n=20)$.

\begin{tabular}{ll}
\hline Parameter & mean \pm SD \\
\hline Age (year) & $23.60 \pm 3.49$ \\
Weigh $(\mathrm{kg})$ & $61.70 \pm 4.32$ \\
Height $(\mathrm{m})$ & $162.90 \pm 6.20$ \\
BMI $\left(\mathrm{kg} / \mathrm{m}^{2}\right)$ & $23.77 \pm 1.88$ \\
Body fat percent & $30.32 \pm 4.65$ \\
Borg perceived fatigue $(0-10)$ & $7.9 \pm 0.6$ \\
\hline
\end{tabular}

$\mathrm{BMI}=$ body mass index; BF\%= Body fat percent

Repeated-measures ANOVA analysis used for assessment (espresso Caffeine $=90.10 \pm 6.19$, Decaffeinated coffee $=91.00 \pm 5.67$, Without Caffeine consumption $=74.40 \pm 4.99, \mathrm{~F}=48.04, \mathrm{p}=0.00$ ). Information on espresso coffee, decaffeinated coffee and without coffee effects on $2000 \mathrm{~m}$ dragon boat are conditions presented in Table 2.

Table2. Coffee effect on aerobic power in 2000m dragon boat paddling ( $N=20)$.

\begin{tabular}{llllll}
\hline & Mauchly's W & Mean \pm SD & F & Df & Sig \\
\hline Mauchly's Test & 0.13 & & & 2 & $0.00^{*}$ \\
Without coffee & & $74.40 \pm 4.99$ & & & $0.00^{*}$ \\
Espresso coffee & & $90.10 \pm 6.19$ & 48.04 & 1.07 & \\
Decaffeinated coffee & & $91.00 \pm 5.67$ & & & \\
\hline
\end{tabular}

Without coffee=pre-test; $\mathrm{P} \leq 0.05$

Bonferroni post hock test was used for between group interaction and results indicated significant differences in espresso coffee vs. without coffee group and decaffeinated coffee vs. without coffee group. Although there was no significant difference between espresso coffee compare decaffeinated coffee group (Table3).

Table3. Bonferroni post hoc tests $(\mathrm{N}=20)$.

\begin{tabular}{llll}
\hline Group & & Mean difference & Sig \\
\hline without coffee & Espresso coffee & -15.70 & $0.00^{*}$ \\
without coffee & Decaffeinated coffee & -16.60 & $0.00^{*}$ \\
Espresso coffee & Decaffeinated coffee & -0.90 & 0.51 \\
\hline$P \leq 0.05$ & &
\end{tabular}

$P \leq 0.05$

\section{Discussion}

The current study determined the effects of high dose coffee (espresso coffee) on 2000m dragon boat women paddler compared to decaffeinated coffee or placebo. Numerous studies had been shown the effectiveness of caffeine on improve endurance exercise [6-9, 17]. Interestingly, current study showed that using coffee espresso (high dos coffee) on hour prior the exercise could significantly improve the VO2max compared with without coffee condition.

In support, most studies have shown this effect on aerobic endurance. Graham et al. (1995), examined the exercise responses of well-trained endurance athletes to various doses of caffeine $(3,6$ and $9 \mathrm{mg} / \mathrm{kg}) \mathrm{one}$ hour before ran at $85 \%$ of maximal $\mathrm{O} 2$ consumption to voluntary exhaustion and showed that endurance was enhanced with both 3 and $6 \mathrm{mg} / \mathrm{kg}$ of caffeine, whereas there was no significant effect with $9 \mathrm{mg} / \mathrm{kg}$ of caffeine. In contrast 
plasma epinephrine was increased in 6 and $9 \mathrm{mg} / \mathrm{kg}$. Similarly only the highest dos of caffeine resulted in increases in glycerol and free fatty acids. Thus, the highest dose had the greatest effect on epinephrine and blood-borne metabolites yet had the least effect on performance [16]. Caffeine acts as phosphodiesterase (PDE) inhibitor that causes an increase in cyclic adenosine monophosphate (cAMP) in the brain and leads to stimulation of catecholamines (i.e., epinephrine) [25, 26]. This elevation in epinephrine levels [27] would, in turn, improve performance by delaying carbohydrate utilization and preserving muscle glycogen content and it Cause a greater stimulation of lipolysis during exercise and a greater release of circulating FFAs into the blood stream [28]. Also this potential performance-enhancing mechanism may be particularly relevant for liver (Bischof et al. 2001) glycogen stores [29]. Spriet et al. (1992) found that ingestion of a high dose of caffeine (9mg/kg) before cycled to exhaustion at approximately $80 \%$ maximal oxygen uptake (VO2max) reduced muscle glycogenolysis in the initial 15 min of exercise by increasing free fatty acid (FFA) levels which inhibits glycolysis and spares glycogen for later use [11]. Also Costill and colleagues (1978) observed with their pioneering work on coffee consumption with caffeine $(330 \mathrm{mg})$ in elite male and female cyclists. During exercise, this greater reliance on fat metabolism with caffeine intake spares muscle glycogen utilization, which likely limits fatigue during prolonged aerobic activities (30). Specifically, Essig et al. (1980) and Erickson et al. (1987) both reported 42\% reductions in muscle glycogen use during cycling at $70 \%$ of maximal oxygen uptake following caffeine ingestion compared with a controlplacebo [31].

Lamina et al. (2009) in a study on twenty non-athletes and non-regular users of caffeine African yang male in Different doses $(5,10 \& 15 \mathrm{mg} / \mathrm{kg})$ of caffeine, indicated no ergogenic effect of caffeine on VO2max in short maximum endurance performance [15]. Graham et al. (1991) showed that a high caffeine dose of caffeine $(9 \mathrm{mg} / \mathrm{kg})$ or placebo one hour pre-exercise at approximately 85\% VO2max; two trials running to exhaustion and two trials cycling to exhaustion had no effect on epinephrine concentration was with caffeine before running and cycling. Plasma glycerol was elevated only at exhaustion during cycling and Urinary increased after the running and cycling trials [32]. It seems that, difference result from different performance tests. Lamina et al used short maximum endurance, Graham et al. used a two trials running or cycling to exhaustion (85\% VO2max) and we used $2000 \mathrm{~m}$ paddling.

Also, this study demonstrated the effectiveness of decaffeinated coffee on improve VO2max compare with without coffee condition. Hodgson et al (2013) illustrated performance times during the time trail were significantly faster for both caffeine and coffee when compared to placebo and decaffeinated coffee performance, they were similar for both caffeine and coffee and no significant differences were observed between placebo and decaffeinated coffee during the time trail in eight trained male cyclists/triathlon athletes following 30 min of steady-state cycling at approximately 55\% VO2max and consumption drinks consisting $5 \mathrm{mg} / \mathrm{kg}$ of caffeine, instant coffee, instant decaffeinated coffee or placebo [8]. In a study by Graham et al. (1998), nine healthy, fit, young adults performed five trials after ingesting placebo (dextrose) capsules with water, caffeine capsules with water, regular coffee, decaffeinated coffee, or decaffeinated coffee plus caffeine. In all three Caffeine trials, the Caffeine dose was $4.45 \mathrm{mg} / \mathrm{kg}$. After one hour of rest, the subject ran at $85 \%$ of maximal $\mathrm{O} 2$ consumption until voluntary exhaustion. The results showed plasma Caffeine and epinephrine was increased in the three Caffeine trials. But the plasma epinephrine increase was greater with Caffeine capsules than with Coffee and they suggested that there must be a component (s) of Coffee that moderates the actions of Caffeine [19].

In conclusion, caffeine can improve performance during aerobic exercise with low or moderate doses of caffeine. Furthermore, coffee can be effectiveness for enhance performance. The present study shows that high doses of caffeine can enhance VO2max during aerobic exercise including $2000 \mathrm{~m}$ dragon boat paddling. Decaffeinated coffee could also have this effect. It seems that some compounds except caffeine in decaffeinated coffee can act improve aerobic power. A limitation of this study was the low number of volunteers obtained because of the total number of Club members was low. Also, subject habit in coffee consumption was not assessed. Further studies needed for considering the effect of high doses of coffee on endurance exercises. In addition more study needed for other age ranges of women athletes and other sport athletes.

\section{Acknowledgements}

This project was supported by Islamic Azad university of Rasht, Iran.

\section{Conflict of interests}

The authors declare that there is no conflict of interests. 


\section{References}

1. Davies RC, Eston RG, Poole DC, Rowlands AV, DiMenna F, Wilkerson DP, et al. Effect of eccentric exerciseinduced muscle damage on the dynamics of muscle oxygenation and pulmonary oxygen uptake. $J$ Appl Physiol, 2008; 105 (5):1413-1421.

2. Singh R, Singh HJ, Sirisinghe RG. Physical and physiological profiles of Malaysian dragon boat rowers. $\mathrm{Br}$ J Sports Med., 1995;29 (1):13-15.

3. Grembecka M, Malinowska E, Szefer P. Differentiation of market coffee and its infusions in view of their mineral composition. Science of The Total Environment, 2007;383 (1-3):59-69.

4. Vignoli JA, Bassoli DG, Benassi MT. Antioxidant activity, polyphenols, caffeine and melanoidins in soluble coffee: The influence of processing conditions and raw material. Food Chemistry, 2011;124 (3):863-868.

5. Doherty M, Smith PM. Effects of caffeine ingestion on exercise testing: a meta-analysis. Int J Sport Nutr Exerc Metab. 2004;14 (6):626-646.

6. Ganio MS, Klau JF, Casa DJ, Armstrong LE, Maresh CM. Effect of Caffeine on Sport-Specific Endurance Performance: A Systematic Review. The Journal of Strength \& Conditioning Research, 2009;23 (1):315-324.

7. Skinner TL, Jenkins DG, Coombes JS, Taaffe DR, Leveritt MD. Dose response of caffeine on 2000-m rowing performance. Medicine and science in sports and exercise, 2010;42 (3):571-576.

8. Hodgson AB, Randell RK, Jeukendrup AE. The metabolic and performance effects of caffeine compared to coffee during endurance exercise. PLoS One, 2013;8 (4):555-561.

9. Ali A, O’Donnell J, Von Hurst P, Foskett A, Holland S, Starck C, et al. Caffeine ingestion enhances perceptual responses during intermittent exercise in female team-game players. Journal of Sports Sciences, 2015;1:1-12.

10. Fredholm BB, Battig K, Holmen J, Nehlig A, Zvartau EE. Actions of caffeine in the brain with special reference to factors that contribute to its widespread use. Pharmacological reviews, 1999;51 (1):83-133.

11. Spriet LL, MacLean DA, Dyck DJ, Hultman E, Cederblad G, Graham TE. Caffeine ingestion and muscle metabolism during prolonged exercise in humans. The American journal of physiology, 1992;262 (6):891898.

12. Rush JWE, Spriet LL. Skeletal muscle glycogen phosphorylase akinetics: effects of adenine nucleotides and caffeine. Journal of Applied Physiology, 2001;91 (5):2071-2078.

13. Black CD, Waddell DE, Gonglach AR. Caffeine's Ergogenic Effects on Cycling: Neuromuscular and Perceptual Factors. Medicine and science in sports and exercise, 2015;47 (6):1145-1158.

14. Bortolotti H, Altimari LR, Vitor-Costa M, Cyrino ES. Performance during a 20-km cycling time-trial after caffeine ingestion. J Int Soc Sports Nutr. 2014;11:45-50.

15. Lamina S, Musa DI. Ergogenic effect of varied doses of coffee-caffeine on maximal aerobic power of young African subjects. African Health Sciences. 2009;9 (4):270-274.

16. Graham TE, Spriet LL. Metabolic, catecholamine, and exercise performance responses to various doses of caffeine. Journal of applied physiology, 1995;78 (3):867-874.

17. Desbrow B, Leveritt M. Awareness and use of caffeine by athletes competing at the 2005 Ironman Triathlon World Championships. Int J Sport Nutr Exerc Metab. 2006;16 (5):545-558.

18. McLellan TM, Bell DG. The impact of prior coffee consumption on the subsequent ergogenic effect of anhydrous caffeine. Int J Sport Nutr Exerc Metab. 2004 Dec;14 (6):698-708.

19. Graham TE, Hibbert E, Sathasivam P. Metabolic and exercise endurance effects of coffee and caffeine ingestion. J Appl Physiol, 1998; 85 (3):883-889.

20. Plaskett CJ, Cafarelli E. Caffeine increases endurance and attenuates force sensation during submaximal isometric contractions. J Appl Physiol, 2001; 91 (4):1535-1544.

21. Borg GA. Psychophysical bases of perceived exertion. Med sci sports exerc. 1982;14 (5):377-381.

22. Lohman TG, Roche AF, Martorell R. Anthropometric standardization reference manual. Champaign, IL: Human Kinetics Books; 1988.

23. Welcome to KayakPro Europe Direct. Available at: http://www.kayakpro.com/ (accessed 23.07.2016).

24. Graham TE. Caffeine and exercise: metabolism, endurance and performance. Sports Med. 2001;31 (11):785807.

25. Graham TE, Battram DS, Dela F, El-Sohemy A, Thong FSL. Does caffeine alter muscle carbohydrate and fat metabolism during exercise? Applied Physiology, Nutrition, and Metabolism, 2008;33 (6):1311-1318.

26. Fredholm BB. On the mechanism of action of theophylline and caffeine. Acta medica Scandinavica. 1985;217 (2):149-153.

27. Greer F, Friars D, Graham TE. Comparison of caffeine and theophylline ingestion: exercise metabolism and endurance. J Appl Physiol, 2000;89 (5):1837-1844. 
28. Ryu S, Choi S-K, Joung S-S, Suh H, Cha Y-S, Lee S, et al. Caffeine as a Lipolytic Food Component Increases Endurance Performance in Rats and Athletes. Journal of Nutritional Science and Vitaminology, 2001;47 (2):139-146.

29. Bischof MG, Krssak M, Krebs M, Bernroider E, Stingl H, Waldhäusl W, et al. Effects of short-term improvement of insulin treatment and glycemia on hepatic glycogen metabolism in type 1 diabetes. Diabetes, 2001;50 (2):392-398.

30. Costill DL, Dalsky GP, Fink WJ. Effects of caffeine ingestion on metabolism and exercise performance. Med Sci Sports. 1978;10 (3):155-158.

31. Essig D, Costill D, Van Handel P. Effects of caffeine ingestion on utilization of muscle glycogen and lipid during leg ergometer cycling. Int J Sports Med, 1980;01 (2): 86-90. doi:10.1055/s-2008-1034637

32. Graham TE, Spriet LL. Performance and metabolic responses to a high caffeine dose during prolonged exercise. Journal of applied physiology, 1991;71 (6):2292-2298.

\begin{tabular}{|c|}
\hline Information about the authors: \\
\hline $\begin{array}{l}\text { Ramin Shabani; http://orcid.org/0000-0002-2681-3814; } \\
\text { shabani_msn@yahoo.com; Islamic Azad University; PO Box: } \\
\text { 8514143131, Rasht, Iran. }\end{array}$ \\
\hline $\begin{array}{l}\text { Roya Rahmatian; http://orcid.org/0000-0003-2974-7207; } \\
\text { fa.izaddoust@gmail.com; Islamic Azad University; PO Box: } \\
\text { 8514143131, Rasht, Iran. }\end{array}$ \\
\hline $\begin{array}{l}\text { Fatemeh Izaddoust; http://orcid.org/0000-0001-9680-6364; } \\
\text { fa.izaddoust@gmail.com; Islamic Azad University; PO Box: } \\
\text { 8514143131, Rasht, Iran. }\end{array}$ \\
\hline $\begin{array}{l}\text { Cite this article as: Ramin Shabani, Roya Rahmatian, } \\
\text { Fatemeh Izaddoust. Effects of high dose coffee intake on } \\
\text { aerobic power in dragon female athletes. Physical education }\end{array}$ \\
\hline 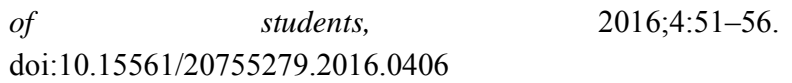 \\
\hline $\begin{array}{l}\text { The electronic version of this article is the complete one and can be } \\
\text { found online at: http://www.sportpedu.org.ua/html/arhive-e.html }\end{array}$ \\
\hline $\begin{array}{l}\text { This is an Open Access article distributed under the terms of the } \\
\text { Creative Commons Attribution License, which permits unrestricted } \\
\text { use, distribution, and reproduction in any medium, provided the } \\
\text { original is is properly } \\
\text { (http://creativecommons.org/licenses/by/4.0/deed.en). }\end{array}$ \\
\hline $\begin{array}{l}\text { Received: } 28.07 .2016 \\
\text { Accepted: } 19.08 .2016 ; \mathrm{Pu}\end{array}$ \\
\hline
\end{tabular}

